\title{
Preprints in biology
}

We remind our readers about our policies on the use of preprints: in short, we support them. A Nature Methods author can post a preprint prior to submission without fearing a penalty.

Most physics papers published in Nature journals begin as preprints-manuscripts that scientists disseminate to their peers, typically via a preprint server, before they have been formally peer reviewed. This reflects the status quo in physics and computer science, where preprints have been widely used for decades, even before the popular preprint server arXiv was created in 1991. Preprints have yet to be embraced, however, by most biologists.

Earlier this year, proponents of the use of preprints in biology gathered to discuss how this form of manuscript may be used to improve the dissemination of biological research results. The ASAPbio 2016 meeting was also attended by representatives of the Nature journals, as well as by major funders and other publishers.

The policy of Nature Methods and all other Nature journals regarding preprints is unambiguous: we welcome the posting of preprints on accepted preprint servers such as bioRxiv and arXiv, or on an author's website or blog. Our full policy, including on press embargo, is here. Indeed, Nature Publishing Group launched its own preprint server, Nature Precedings, in 2007, although it is no longer active. At Nature Methods, we find that within certain fields-genomics and computational biology in particular-submitted papers are quite commonly first posted as preprints. In line with our policy, this does not affect their consideration, review, or publication in this journal. We note that not all publishers consider the posting of preprints to be acceptable; researchers should inform themselves of the policies of their preferred publication venue.

Both by our own qualitative estimate and according to the results of a survey conducted prior to ASAPbio 2016, most biologists do not submit preprints at the moment. ASAPbio found that about one-third of biologists who responded to its survey had submitted preprints; the group also recognized that this is probably not representative of the whole community, as the survey is likely to have selected for scientists already attuned to the practice.

Posting a preprint means that scientists' work is rapidly distributed to their peers before publication. In the best case, it improves communication and scientific discourse. It could directly improve manuscripts, as critique from interested peers could be incorporated. But in many fields the fear of being 'scooped' is real: witness the dearth of presentations of unpublished work at many biology conferences. Although the preprint is typically a more developed version of research results and should therefore be less susceptible to such problems, it is likely that preprints will not become widely accepted in biology unless, like in physics, they serve as a communityaccepted mark of intellectual priority.

Separately, a preprint is an interim, informal record of a scientist's work; given that peer review and formal publication are relatively slow processes, they could more nimbly be incorporated into applications for funding renewal, faculty positions, or promotions. For them to be effective in this way, though, funders and hiring committees would have to accept preprints as credible even though they have not been peer reviewed, or (more responsibly) they would have to assess the scientific credibility of preprints as part of their decision process.

Manuscripts frequently change over the course of peer review. It is not uncommon, for example, for a paper at Nature Methods to be declined because the referees are not convinced that the method measures accurately and robustly what it claims to measure. It is even more common that, after peer review, inflated claims in a paper are qualified or modified in some substantial way. Posting one or more preprints prior to publication would mean that a manuscript would exist in several versions that could differ in their interpretation. Responsible adoption of the preprint needs robust versioning and a mechanism to link to the reviewed, published paper, as provided by bioRxiv.

As for any scientific work, there is the potential for misinterpretation or even misrepresentation of preprints by non-experts. In biology in particular, where links to human disease and claims of medical relevance are frequently made, the responsibility to the public of making a genuine effort to vet these claims is of the highest order. Preprints, like unpublished work presented at conferences, must state that claims have not yet been peer reviewed. We note that Nature's policy requires that authors not solicit media attention to posted preprints.

Preprints enable the rapid dissemination of biological research results, an inarguable good. Their widespread use could also affect how credit is assigned and scientific output is assessed, and could make unvetted claims prematurely accessible to the wider public; these and other consequences should be reflected upon by the biological research community. Cultural differences between the biological and physical sciences are likely to provide fodder for fertile thought. We encourage our readers to participate in this discussion. 